Check for updates

Cite this: Mater. Adv., 2022, 3,547

Received 7th October 2021,

Accepted 7th November 2021

DOI: 10.1039/d1ma00931a

rsc.li/materials-advances

\title{
External complexation of BODIPYs by CB[7] improves in-cell fluorescence imaging $\dagger$
}

\author{
Mehmet Menaf Ayhan, (D) *a Emrah Özcan, ${ }^{a b}$ Fahri Alkan, (D) c Metin Çetin, ${ }^{d}$ ilker Ün, ${ }^{e}$ \\ David Bardelang (iD ${ }^{f}$ and Bünyemin Çoşut (iD *a
}

\begin{abstract}
Organic luminescent compounds with high emission properties play a crucial role in fluorescence labelling and optoelectronic devices. In this work, we prepared three water soluble BODIPY derivatives (B-4, B-5, and B-6) which are weakly fluorescent due to non-radiative relaxation pathways (charge transfer: $\mathrm{CT}$ or heavy atom effect). However, CB[7] significantly improves BODIPY fluorescence by $\sim 10$ fold for B-4, and by $\sim 3$ fold for B-5. The (TD)DFT analyses suggest that for B-4 and B-5, the CT state is blue-shifted as a result of the external binding of CB[7] near the pyridinium groups. This effect favoured a radiative decay through a locally-excited (LE) $\pi \rightarrow \pi^{*}$ transition state of BODIPYs resulting in a CB[7]-induced emission increase in solution (and in the solid state), without compromising singlet-totriplet intersystem crossing (ISC). The improved emission of the BODIPY.CB[7] complexes was used for the fluorescence imaging of U87 cells illustrating the relevance of this approach. These results suggest that BODIPY.CB[7] complexes could be used as theragnostic agents by combining fluorescence imaging and treatment by photodynamic therapy.
\end{abstract}

\section{Introduction}

Boron dipyrromethene (BODIPY) derivatives are one of the most actively studied groups of fluorophores due to the ease of synthesis, excellent photostability, and tunability of their photophysical and photochemical properties. These organic fluorophores are attracting a great deal of attention because they have been used in solar cells, ${ }^{1}$ photodynamic therapy (PDT) ${ }^{2,3}$ organic fluorescent emitting devices (OFEDs), ${ }^{4,5}$ semiconductors, ${ }^{6}$ bioimaging, ${ }^{7-9}$ and chemo-sensors. ${ }^{10}$ According to the Jablonski diagram, the excitation of a BODIPY chromophore leads to three competitive pathways of energy emission corresponding to different photo-functions: fluorescence emission $(\mathrm{S} 1 \rightarrow \mathrm{S} 0$ radiative decay), intersystem crossing or ISC (S1 $\rightarrow \mathrm{T} 1$ non-radiative transition), and other nonradiative decay pathways (e.g. rotation, distortion, vibration, or charge transfer (CT)).

\footnotetext{
${ }^{a}$ Department of Chemistry, Gebze Technical University, Gebze, Kocaeli, Turkey

${ }^{b}$ Institute of Physics, Faculty of Science, University of South Bohemia, Branišovská 1760, 370 05, České Budějovice, Czech Republic. E-mail: bcosut@gtu.edu.tr, menafayhan@gtu.edu.tr

${ }^{c}$ Department of Nanotechnology Engineering, Abdullah Gül University, Kayseri, Turkey

${ }^{d}$ Department of Molecular Biology and Genetics, Gebze Technical University, Gebze, Kocaeli, Turkey

${ }^{e}$ Chemistry Group Laboratories, TÜBİTAK UME, Gebze, Kocaeli, Turkey

${ }^{f}$ Aix Marseille Univ, CNRS, ICR, Marseille, France

$\dagger$ Electronic supplementary information (ESI) available. CCDC 2051939. For ESI and crystallographic data in CIF or other electronic format see DOI: 10.1039/ d1ma00931a
}

Optimization of a given photo-function can be performed by suppressing undesired decay pathways. Moreover, BODIPYs often tend to aggregate in polar solvents causing decrease in emission and thereby limiting their use in biological applications. In the past decade, significant efforts were devoted to develop strategies aiming to address these drawbacks and improve the photophysical features of BODIPYs. ${ }^{11}$ Current approaches mainly focus on chemically modifying BODIPYs to suppress aggregation and nonradiative decay pathways, but require specific structural designs and sizable synthetic efforts. ${ }^{12}$ Besides, aggregation-induced emission (AIE) has recently emerged as another way to obtain useful fluorescence; however, this method is limited by the small number of AIE chromophores, the challenge of controlling aggregation, ${ }^{11,13-16}$ and the concerns of colloidal stability questioning safety for biomedical applications. Alternatively, supramolecular chemistry could afford low-cost strategies to tune the photophysical properties of BODIPYs by forming host-guest inclusion complexes. ${ }^{17-21}$ For biological applications, water-soluble host molecules are required. Among macrocyclic compounds, ${ }^{22,23}$ cucurbiturils $(\mathrm{CB}[n]: n=5-8,10)^{24-26}$ occupy a special position due to the availability of various cavity sizes, their rigid hydrophobic cavity lined by two carbonyl fringed portals and their low toxicity. ${ }^{25,27-30}$ Among the $\mathrm{CB}[n]$ macrocycles, $\mathrm{CB}[7]$ (Chart 1) has received a lot of attention due to its reasonably good water solubility, appropriate cavity size for most therapeutic agents and conventional chromophores, ${ }^{31-36}$ and low toxicity. ${ }^{37}$ Several researchers have helped to push forward the field of fluorescent dyes by making key 


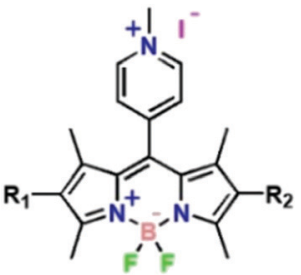

BODIPYs

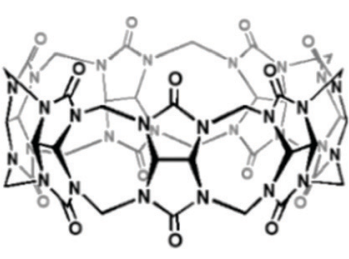

cucurbit[7]uril
Chart 1 Structures of BODIPYs and CB[7] used in this work (R1, R2 = H or I).

contributions on supramolecular complexes involving macrocycles, in particular $\mathrm{CB}[n] .{ }^{18}$ Several classes of dyes have thus received intense attention and often fluorescence increases are noted after $\mathrm{CB}[n]$ encapsulation due to disfavored non radiative decay processes, deaggregation, and dyes experiencing both low polarity and low polarizability cavities. ${ }^{38}$ However, today, there are only a handful of studies about the effect of cucurbiturils on the photophysical and photochemical properties of BODIPY molecules. ${ }^{39-46}$ In most cases, cucurbituril encapsulation increases fluorescence by either preventing aggregation or manipulating the guest $\mathrm{p} K_{\mathrm{a}}$ values. In this work, we used $\mathrm{CB}[7]$ to inhibit the nonradiative decay processes of excited BODIPYs by external complexation enabling to improve guest emission for fluorescence imaging. The "supra-chromophore" approach enabled the number of synthetic steps to be dramatically reduced while allowing switchable photophysical properties to be introduced.

\section{Results and discussion}

\section{Experimental}

Synthetic procedures. For this purpose, we have prepared BODIPY derivatives (B-4, B-5, and B-6, Scheme 1) in a few synthetic steps, affording water soluble chromophores by means of their cationic groups which are also designed to complex well with $\mathrm{CB}[7]$.

B-1, B-2, and B-3 were synthesized according to the literature procedures. ${ }^{47}$ Next, B-1, B-2, and B-3 underwent a reaction with 20 equivalents of methyl iodide in acetonitrile at $40{ }^{\circ} \mathrm{C}$. The reaction proceeded smoothly in $24 \mathrm{~h}$ with full conversion and afforded the targets B-4, B-5, and B-6 in $\sim 75 \%$ isolated yield. Incorporation of the methyl group was supported by the presence of a diagnostic peak at $4.77 \mathrm{ppm}$ in their ${ }^{1} \mathrm{H}$ NMR spectra (Fig. S1, S3, and S5, ESI $\dagger$ ). ${ }^{13} \mathrm{C}$ NMR spectra and mass spectra confirmed this result (Fig. S2, S4, S6, S7, S8 and S9, ESI $\dagger$ ). Additionally, the molecular structure of B-4 was further established by single-crystal X-ray diffraction (vide infra, Fig. 4a and Table S1, ESI $\dagger$ ).

Photophysical properties. The optical features of B-4, B-5, and B-6 in the absence and in the presence of $\mathrm{CB}[7]$ were studied by UV-Vis absorption, fluorescence, and time resolved fluorescence spectroscopies. As shown in Fig. S10 (ESI $\dagger$ ), B-4, B-5, and B-6 show absorption maximum at $505 \mathrm{~nm}, 525 \mathrm{~nm}$,

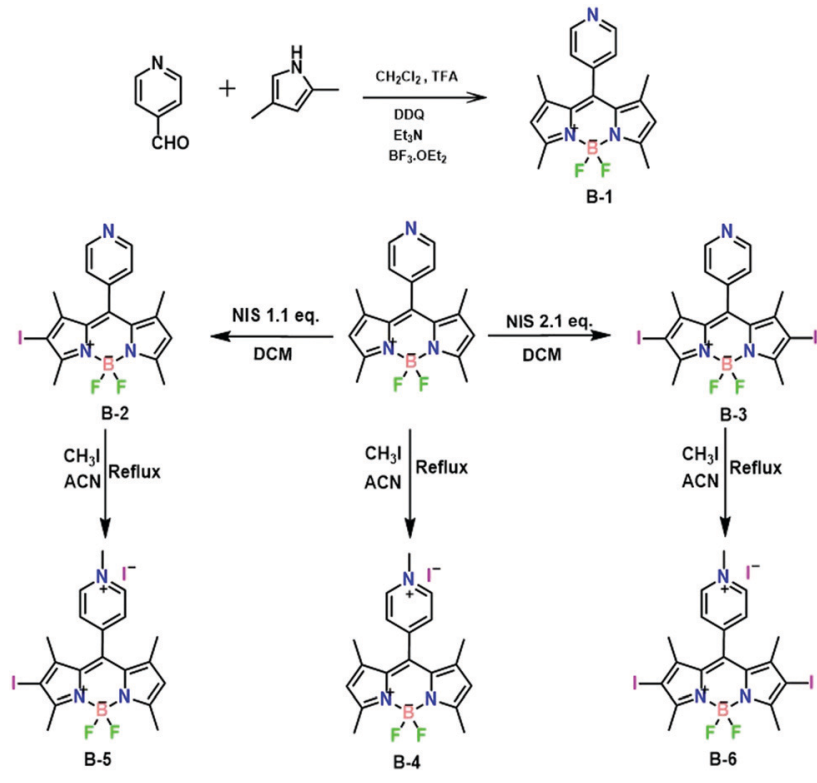

Scheme 1 Synthetic route to BODIPYs B-4, B-5, and B-6.
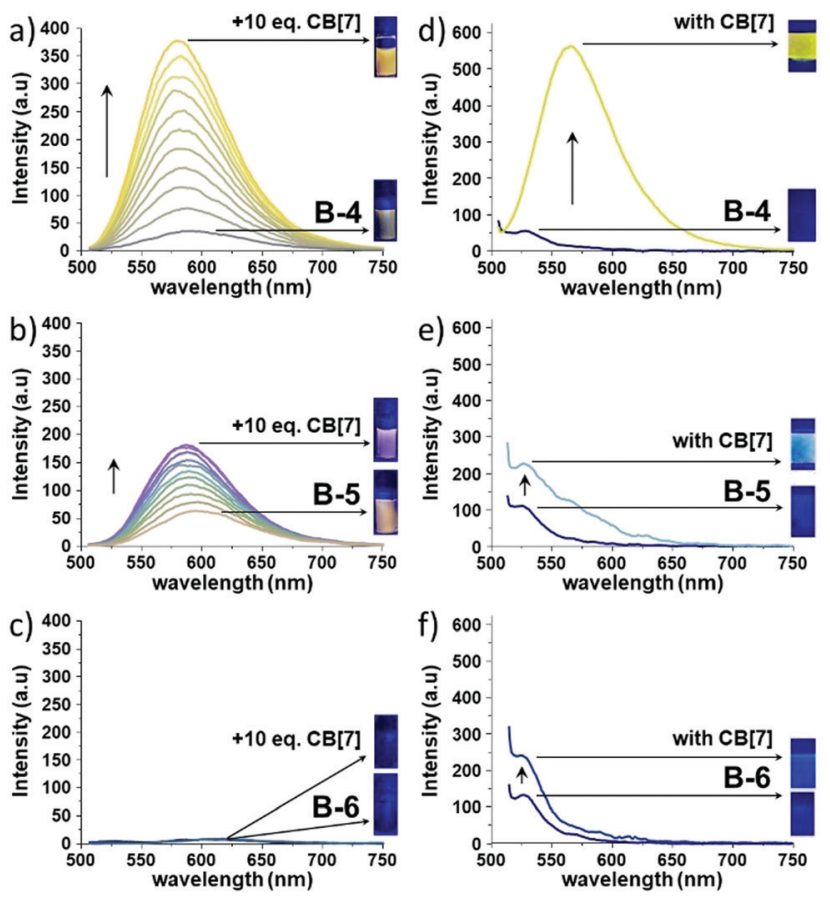

Fig. 1 Fluorescence spectra of (a) B-4, (b) B-5, and (c) B-6 $\left(1.0 \times 10^{-5} \mathrm{M}\right.$ each, excitation wavelength $=490 \mathrm{~nm}$ ) in water upon the addition of CB[7] and solid state fluorescence spectra of (d) B-4, (e) B-5, and (f) B-6 without and with $\mathrm{CB}[7]$.

and $545 \mathrm{~nm}$, respectively. These absorption profiles are specific for meso-aromatic BODIPYs and assigned to $\pi \rightarrow \pi^{*}$ transitions $(\mathrm{S} 0 \rightarrow \mathrm{S} 1){ }^{48}$ The absorption maximum for $\mathbf{B}-\mathbf{5}$ and B-6 is significantly red-shifted compared to that of B-4 owing to the presence of one (or two) iodine atom(s), respectively. B-4, B-5, and B-6 are weakly emissive. Fluorescence spectra show a weak intensity around $590 \mathrm{~nm}$ (Fig. 1 left column and Fig. S11, ESI $\dagger$ ) 

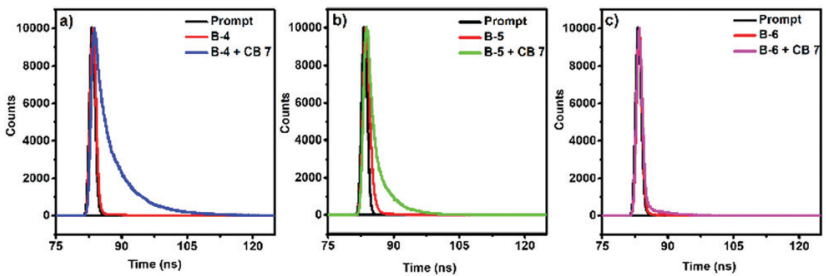

Fig. 2 Fluorescence decay profiles of (a) B-4 without and with $C B[7]$ (b) B-5 without and with CB[7], and (c) B-6 (10-5 M) without and with CB[7] using laser excitation at $390 \mathrm{~nm}$.

a)
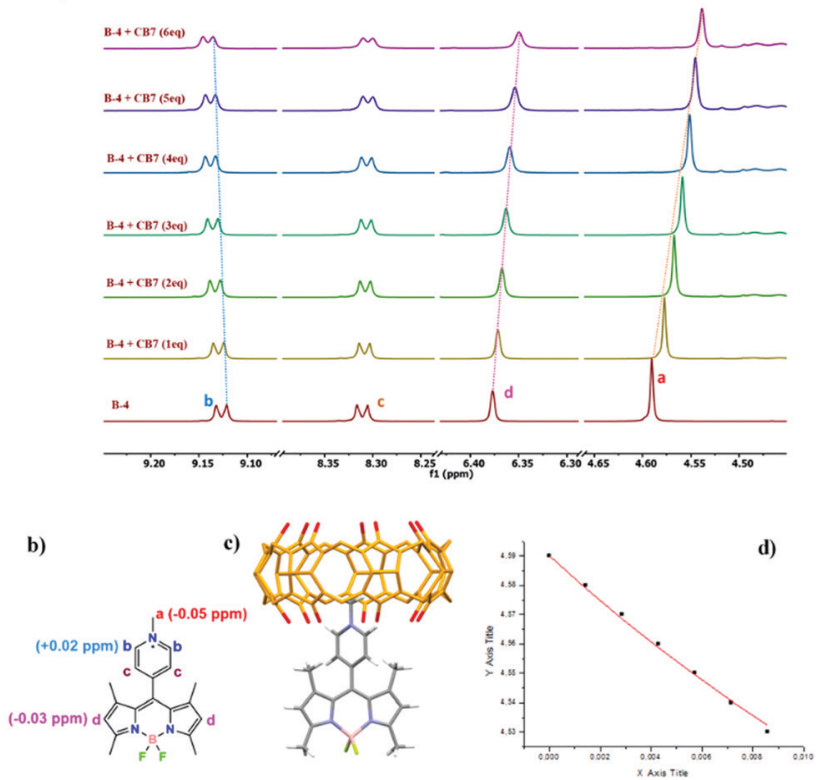

Fig. 3 (a) ${ }^{1} \mathrm{H}-\mathrm{NMR}$ titration of $\mathbf{B}-\mathbf{4}(0.5 \mathrm{mM})$ with $\mathrm{CB}$ [7] in $\mathrm{D}_{2} \mathrm{O} / \mathrm{CD}_{3} \mathrm{CN}$ $(80: 20)$ solution, (b) assignment of the corresponding proton chemical shifts and (c) lowest energy DFT minimized structure of the 1:1 inclusion complex of B-4 + CB [7]. (d) The chemical shift of guest molecule Ha versus $\mathrm{CB}[7]$ concentration and the non-linear curve fit in red.

especially for B-5 and B-6 mainly caused by halogen atoms. These atoms are known to induce intersystem crossing via strong spin-orbit coupling between the singlet and triplet states, the so-called heavy-atom effect. ${ }^{49,50}$

Upon $\mathrm{CB}[7]$ addition, the absorption profiles of $\mathbf{B}-\mathbf{4}, \mathbf{B}-\mathbf{5}$, and B-6 remained nearly identical $(\Delta \lambda \max \sim 3-4 \mathrm{~nm})$ in line with the absence of $\mathrm{CB}[7]$-induced deaggregation and thus suggesting that the BODIPYs are soluble at $\mu \mathrm{M}$ concentrations (Fig. S10, ESI $\dagger$ ). In stark contrast, the corresponding fluorescence spectra showed dramatic changes after $\mathrm{CB}$ [7] addition (Fig. 1, left column and Fig. S11, ESI $\dagger$ ). The fluorescence intensity of B-4 was enhanced by $\sim 10$ fold and blue-shifted by $13 \mathrm{~nm}$ in the presence of 10 equivalents of $\mathrm{CB}[7]$. Similarly, the fluorescence intensity of B-5 increased by $\sim 3$ fold, with a slight blue shift by $8 \mathrm{~nm}$ in emission maximum with $\mathrm{CB}[7]$. However, the addition of $\mathrm{CB}[7]$ to an aqueous solution of B-6 did not cause significant changes in guest emission intensity. We should note that, at $10^{-6} \mathrm{M}$ concentration of $\mathbf{B}-\mathbf{4}, \mathbf{B}-\mathbf{5}$, and a)

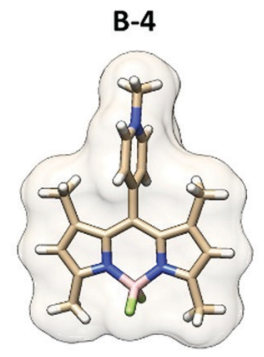

b)

$\mathrm{CB}[7]$

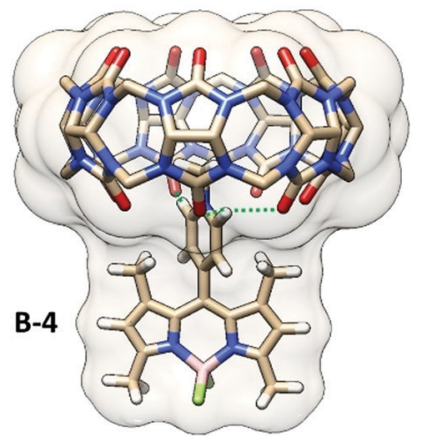

Fig. 4 X-ray structure of B-4 and DFT minimized structure of the $\mathbf{B}-\mathbf{4}$. CB[7] complex (hydrogen bonds: dashed lines, hydrogens of CB[7] removed for clarity).

B-6, the corresponding fluorescence spectra showed only small changes after $\mathrm{CB}$ [7] addition probably due to weak binding interactions (Fig. S12, ESI $\dagger$ ). Furthermore, the solid-state emission properties of B-4, B-5, and B-6 without and with $\mathrm{CB}[7]$ were also explored. Solid films obtained on glass slides by slow solvent evaporation of aqueous solutions of B-4, B-5, and B-6, without and with $\mathrm{CB}[7]$, were analyzed by solid state fluorescence spectroscopy. Very weak emissions were observed at $530 \mathrm{~nm}$, blue-shifted compared solution-state emissions (Fig. 1, right column). The solid-state emission intensity of B-4 and B-5 was decreased compared to the emissions in solution due to aggregation-induced quenching combined with non-radiative pathways. However, $\mathrm{CB}[7]$ caused a significant increase in the solid-state emission of $\mathbf{B}-\mathbf{4}(\sim 9$ fold that of free B-4) and a red shift of $36 \mathrm{~nm}$. Nevertheless, the corresponding spectra for B-5 and B-6 only showed a small and negligible increase in emission intensity, respectively, at the same wavelength in the presence of $\mathrm{CB}[7]$. Besides, $\mathrm{CB}[7]$ has been shown to increase fluorescence lifetimes and quantum yields of the guest molecules due to its low polar inner cavity. Fluorescence decay measurements of B-4, B-5, and B-6 in the absence and presence of $\mathrm{CB}[7]$ are shown in Fig. 2 and Table 1.

Without $\mathrm{CB}[7]$, the fluorescence decay of B-4 and B-5 was mono-exponential, corresponding to fluorescence lifetimes of about 0.18 and $0.60 \mathrm{~ns}$, respectively. However, in the presence of $\mathrm{CB}[7]$, the fluorescence decay rates decreased while showing multi-exponential behaviors with major contributions from a longer lifetime $\left(\tau_{2}\right)$ component of $6.21 \mathrm{~ns}$ for $\mathbf{B}-4$ and $3.84 \mathrm{~ns}$

Table 1 Photophysical properties of B-4, B-5 and B-6 without and with CB[7] in water

\begin{tabular}{llllllll}
\hline Compound & $\begin{array}{l}\lambda_{\mathrm{abs}} \\
(\mathrm{nm})\end{array}$ & $\begin{array}{l}\lambda_{\mathrm{em}} \\
(\mathrm{nm})\end{array}$ & $\begin{array}{l}a_{1} \\
(\%)\end{array}$ & $\begin{array}{l}T_{1}{ }^{a} \\
(\mathrm{~ns})\end{array}$ & $\begin{array}{l}a_{2} \\
(\%)\end{array}$ & $\begin{array}{l}T_{2}{ }^{a} \\
(\mathrm{~ns})\end{array}$ & $\begin{array}{l}\Phi_{\mathrm{F}}{ }^{b} \\
(\%)\end{array}$ \\
\hline B-4 & 505 & 592 & 100 & 0.18 & - & - & 0.6 \\
B-4.CB[7] & 505 & 579 & 25 & 1.15 & 75 & 6.21 & 6 \\
B-5 & 525 & 594 & 100 & 0.60 & - & - & 0.07 \\
B-5·CB[7] & 525 & 586 & 43 & 0.78 & 57 & 3.84 & 1 \\
B-6 & 545 & - & 100 & 0.09 & - & - & - \\
B-6·CB[7] & 545 & - & 100 & 0.17 & - & - & -
\end{tabular}

${ }^{a}$ Lifetime $\left(a_{1} / a_{2}\right.$ : mono-/bi-exponential fitting). ${ }^{b}$ Fluorescence quantum yield. 
for B-5. The presence of $\mathrm{CB}[7]$ has only a weak effect on the fluorescence decay of B-6, being mono-exponential in nature and corresponding to a fluorescence lifetime of $\sim 0.09-0.17 \mathrm{~ns}$. As for the change in quantum yield, the fluorescence quantum yield $\left(\Phi_{\mathrm{F}}\right)$ measurements point to improved fluorescence characteristics with the addition of $\mathrm{CB}[7]$. In the presence of $\mathrm{CB}[7]$, the fluorescence quantum yields of $\mathbf{B}-\mathbf{4}$ and B-5 has increased $\sim 10-13$ fold compared to the absence of $\mathrm{CB}[7]$ (Table 1).

BODIPYs are considered as good photosensitizers for PDT. Singlet oxygen generation measurements for the three BODIPY dyes were then performed in the absence and in the presence of $\mathrm{CB}[7]$ (Fig. S13, ESI $\dagger$ ). While B-4 did not show singlet oxygen generation, B-5 and B-6 efficiently produced singlet oxygen due to the heavy atom effect promoting singlet-to-triplet intersystem crossing (ISC). Surprisingly, CB[7] did not change these results with virtually identical responses with respect to each BODIPY alone. The observed fluorescence improvements without compromising the ISC processes make this supramolecular approach with $\mathrm{CB}[7]$ a promising strategy to develop smart agents combining biological imaging and photodynamic therapy. To clarify the mechanism by which $\mathrm{CB}$ [7] impacts the BODIPY emissive features, we next focused on the structural and theoretical studies.

Structural study. Mass spectrometry of B-4, B-5, and B-6 with and without $\mathrm{CB}[7]$ were performed by MALDI-TOF. The samples were dissolved in water/THF solutions using $\alpha$-cyano-4hydroxycinnamic acid as the matrix. Results support the occurrence of $1: 1$ host: guest complexes for all compounds (Fig. S7, S8, S9, ESI $\dagger$ ). We next used $1 \mathrm{H}$ NMR to get further insights into the kind of supramolecular complex keeping in mind that the BODIPY frame is likely too large to be engulfed in the host cavity. When a guest molecule is included in $\mathrm{CB}$ [7], the signals of the guest protons inside the cavity are shifted upfield $(\Delta \delta<0)$ while the protons sitting next to the $\mathrm{CB}[7]$ carbonyl portals have their signals deshielded compared to those for the guest alone $(\Delta \delta>0) .^{27}$

To ensure full guest solubility at $\mathrm{mM}$ concentrations, we used a small percentage of $\mathrm{CD}_{3} \mathrm{CN}$; this solvent is known to be only weakly competitive $\left(K_{\mathrm{a}, \mathrm{ACN} / \mathrm{CB}[7]}=11 \pm 1 \mathrm{M}^{-1}\right)^{51}$ but also a possible source of weaker complexations. ${ }^{1} \mathrm{H}$ NMR titrations at $0.5 \mathrm{mM}$ of guest and with increasing amounts of $\mathrm{CB}[7]$ in $\mathrm{D}_{2} \mathrm{O}$ / $\mathrm{CD}_{3} \mathrm{CN}$ (80:20 vol\%) showed complexation-induced shifts (CIS) for methyl protons $(\mathrm{Ha})$ of the meso pyridinium group of $\Delta \delta=$ $-0.05,-0.06$, and -0.07 ppm for B-4, B-5, and B-6, respectively (Fig. 3, Fig. S14, S15, ESI $\dagger$ ). This suggests that the pyridinium group of each BODIPY is at least partly included in CB[7]. Small downfield shifts of pyridinium aromatic protons $(\mathrm{Hb})$ support these results, presumably experiencing the deshielding effect of the nearby host carbonyl rim. Simultaneously, the singlets corresponding to pyrrolic hydrogen atoms (Hd) were weakly shielded, probably due to a redistribution of the electron density as signals of the methyl hydrogen atoms of the pyrroles are unchanged. Analysis of the fluorescence spectra of Fig. 1 suggested that the binding constants for equilibria involving the formation of a $1: 1$ complex could be of the order of $10^{3}$ to $10^{4} \mathrm{M}^{-1}$. Non-linear curve fitting using data from NMR titrations $^{52}$ afforded binding constants $K_{\mathrm{a}}, \mathbf{\text { B-4/CB[7] }}=22 \pm$ $11 \mathrm{M}^{-1}, K_{\mathrm{a}},{ }_{\mathbf{B}-5 / \mathrm{CB}[7]}=67 \pm 19 \mathrm{M}^{-1}$, and $K_{\mathrm{a}}$, $\mathbf{\text { B-6/CB} [ 7 ]}=34 \pm$ $16 \mathrm{M}^{-1}$ (Fig. S14, S15, ESI $\dagger$ ), which are rather weak but account for the competitive behavior of acetonitrile necessary to do the titrations. Indeed, even if this co-solvent is weakly competitive, 20 vol\% represent a very large concentration. We hypothesize that binding constants in pure water should be one or two orders of magnitude higher.

To go further, BODIPY B-4 was crystallized, and its singlecrystal X-ray diffraction structure was obtained. The structure showed that the pyridinium group is almost perpendicular to the BODIPY frame because of the restricted rotation imposed by the adjacent methyl groups (Fig. 4a).

While pyridiniums are largely accepted to be good guest fragments for $\mathrm{CB}[7]$, the steric hindrance at the anchor point on the BODIPY frame prevents deep inclusion in the $\mathrm{CB}$ [7] cavity. Indeed, the DFT minimized structure (Fig. 4b), in line with mass spectrometry and NMR shows a shallow inclusion by the methyl-pyridinium moiety, two methyl groups of the BODIPY frame coming close to one carbonyl portal. Three guest:host strong, likely charge-assisted, H-bonds are noticed: one singly engaged $\mathrm{N}^{+}-\mathrm{C}-\mathrm{H} \cdots \mathrm{O}=\mathrm{C}$ bond $(1.90 \AA)$ and a bifurcated $\mathrm{N}^{+}-\mathrm{C}-\mathrm{H} \cdots \mathrm{O}=\mathrm{C}$ bond $(2.38$ and $2.47 \AA$ ). In this geometry, the pyridinium group is slightly rotated off the perpendicular position with respect to the BODIPY frame.

Mechanism of fluorescence enhancement. The electronic structure and photophysical properties of BODIPYs and BODIPY·CB[7] complexes were further investigated using the (TD)DFT methods. Optimized geometries of the ground state showed a torsion angle near perpendicular between the pyridinium moiety and the BODIPY frame suggesting a strong intramolecular donor-acceptor (DA) character with minimal $\pi$-delocalization (Fig. S16, ESI $\dagger$ ). The energy levels from the BODIPY part of B-4 are similar to the corresponding ones of (neutral) B-1, while those from the pyridinium part showed a large stabilization by $1.4-1.7 \mathrm{eV}$. These differences in the electronic structure also reflect on the calculated excited states of B-1 and B-4 (Table S3, ESI $\dagger$ ). In both cases, the S1 level originates from locally-excited (LE) $\pi \rightarrow \pi^{*}$ transitions of BODIPY levels, while dark charge transfer (CT) transition between the $\pi_{\text {BODIPY }} \rightarrow \pi^{*}{ }_{\text {Pyr }} / \pi^{*}$ Pyr+ levels shows a large shift from B-1 to B-4 as expected from their electronic structures (Table S2, ESI $\dagger$ ). This stabilization for the CT state in the cationic system is expected to play an important role in fluorescence quenching. When the excited state geometries of B-4 are optimized, the transition energy of the LE state shows a redshift of $0.35 \mathrm{eV}$ as a result of geometry relaxation (Fig. 5), which is in good agreement with the observed Stokes shift.

The effect of excited-state geometry relaxation on the transition energy of the CT state is even larger $(0.66 \mathrm{eV})$. Overall, both the excited states exhibit similar total energies for their respective minimum. Besides, when the torsion angle $(\theta)$ between the BODIPY and the 4-aryl moieties was changed, pure CT and LE states showed significant mixing to a hybridized state (Fig. S17, ESI $\dagger$ ) pointing to a possible $\mathrm{LE} \rightarrow \mathrm{CT}$ conversion 


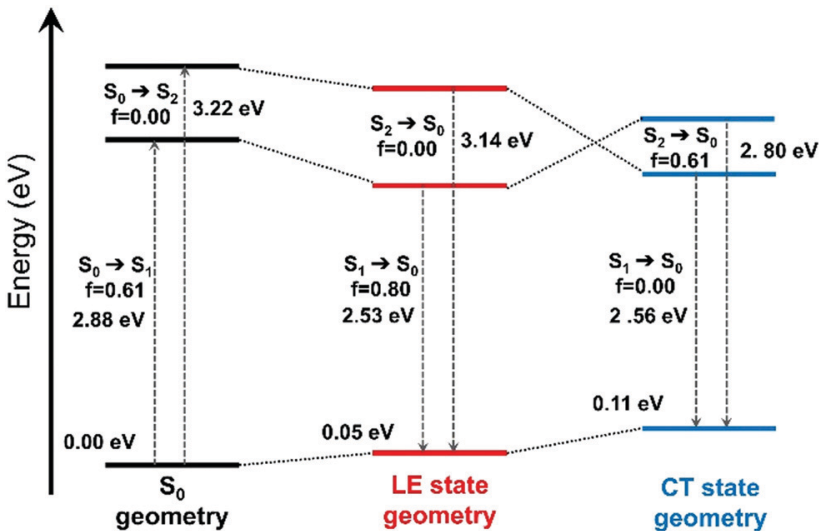

Fig. 5 Evolution of the ground-state and excited-state energies, and of oscillator strengths with respect to different optimized geometries of the ground-state (SO) and the excited-states (LE and CT states) of B-4. The interaction type and MOs involved in these transitions are shown in Fig. S16 (ESI†).

path. These results suggest that the fluorescence quenching observed for B-4 most likely arises from non-radiative relaxation driven by this interplay between the dark CT and bright LE states. ${ }^{53}$ We also note that there is still some overlap between pyridinium and BODIPY-based orbitals for the particle wavefunction as shown in Fig. S17 (ESI $\dagger$ ), which makes complete charge separation and quenching through photoinduced electron transfer (PET) mechanism unlikely for the investigated systems.

The impact of $\mathrm{CB}[7]$ on the excited-state properties of $\mathbf{B}-\mathbf{4}$ was also investigated (Fig. 6). For the B-4.CB[7] complex, both the LE and CT states originate from the same transitions as discussed before, without significant contributions from the $\mathrm{CB}[7]-$ based energy levels. When the distance between B-4 and $\mathrm{CB}[7]$ is large ( $6 \AA$ ), the energy difference between the CT and LE states is predicted to be comparable to the case of the isolated system (Fig. 6c and d, respectively). However, as the distance between $\mathbf{B}-\mathbf{4}$ and $\mathrm{CB}[7]$ decreases, the CT state blueshifts due to ion-dipole interactions between the guest pyridinium moiety and $\mathrm{CB}[7]$, while the energy of the LE state is only marginally affected. For the S0 geometry, the energy difference between the LE and CT states increases to $0.65 \mathrm{eV}$ compared to $0.34 \mathrm{eV}$ for B-4 alone (Fig. S18, ESI $\dagger$ ). For the CT state geometry, $\mathrm{CB}[7]$ complexation swaps energy levels, the LE state becomes the S1 state for distances smaller than $5 \AA$ (Fig. 5d). Beyond the impact on excited-state energetics, $\mathrm{CB}[7]$ complexation is also expected to restrict the guest rotational and vibrational degrees of freedom, thereby limiting the LE $\rightarrow$ CT conversion channel. Consequently, radiative decay from the LE state becomes favorable again for the B-4.CB[7] complex resulting in a significant increase in emission intensity.

Similarly, the excited-state properties were also examined for B-5 and B-6 (Fig. S19, ESI $\dagger$ ). For both BODIPYs, the LE state showed a redshift $(\sim 0.1 \mathrm{eV}$ and $\sim 0.2 \mathrm{eV}$ respectively) in the ground-state geometry compared to that observed for B-4, in line with the experimental results. In comparison, the CT state a)

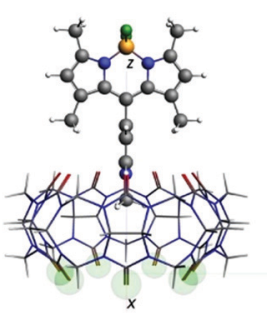

c)

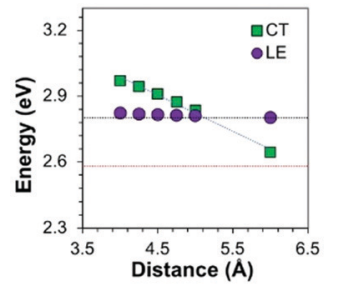

b)

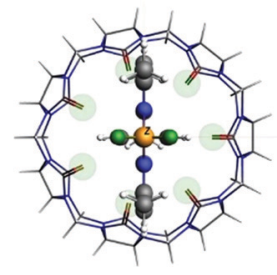

d)

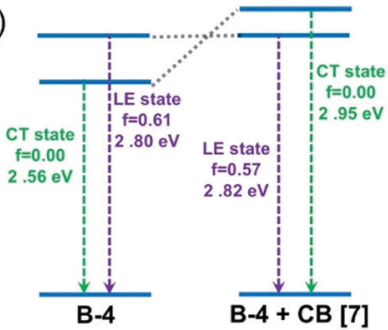

Fig. 6 Different views ( $\mathrm{a}, \mathrm{b}$ ) of the minimized B-4.CB[7] complex. The $\mathbf{B}-4-C B$ [7] distance is plotted in (c) against the energy of the CT and LE states in the optimized CT geometry of B-4. The red and blue dotted lines represent the energies of the CT and LE states, respectively, for isolated B-4 (the distance between CB[7] and B-4 is from the guest carbon atom of the methyl-pyridinium and from the barycenter of the host oxygen atoms; green circles). Energy diagram in (d) illustrates how the energy of the excited-state is impacted by $\mathrm{CB}[7]$ complexation and corresponding to a host-guest distance of $4.25 \AA$ (lowest energy structure for the B-4.CB[7] complex).

showed a blueshift in the range of $\sim 0.25-0.30 \mathrm{eV}$ in the same geometry. Due to LE-state stabilization and CT state destabilization when iodine atoms are introduced, the energy gap between the CT and LE states in both S0 and LE-state geometries increased by $\sim 0.3 \mathrm{eV}$ for B-5 compared to B-4. More importantly, the energetic order of these states does not change for the CT-state geometry. While iodine substitution is expected to increase the fluorescence quenching of B-5 owing to the heavy-atom effect, the electronic structure and excited-states energetics could reduce non-radiative decay from the CT state. In the case of B-6, one would expect a further increase in the emission intensity based solely on excited state energetics as the energy gap between the LE and CT states is even larger. However, the balance becomes unfavorable due to the further iodination of B-6 increasing the ISC rate and resulting in efficient fluorescence quenching in line with previous reports. ${ }^{54}$

Confocal fluorescence imaging. We have shown that the rather counter-intuitive external $\mathrm{CB}[7]$ complexation could improve the fluorescence properties of BODIPY B-4 by alleviating the nonradiative decay pathways. Instilled by these results, we wondered if this supramolecular approach could be used in a more applied context. We thus investigated the relevance of B-4, B-5 and B-6 and of their CB[7] complexes for confocal fluorescence imaging in cells. U87 cells were thus incubated with the BODIPY fluorophores or co-incubated with a BODIPY and $\mathrm{CB}[7]$ (Fig. 7 and Fig. S20, ESI $\dagger$ ).

When U87 cells were incubated with B-4, B-5, or B-6 alone, only a weak fluorescence was observed. However, the fluorescence increased in the presence of $\mathrm{CB}[7]$ and especially when 


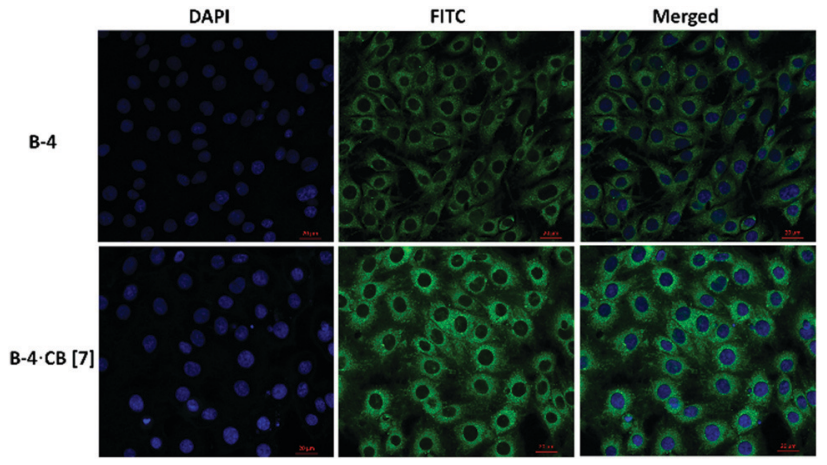

Fig. 7 Confocal fluorescence microscopy images of U87 cells after incubation with B-4 without (top line), and with (bottom line) CB[7] for 4 hours. Cell nuclei were stained with DAPI after PFA fixation (DAPI, $\lambda_{\mathrm{Ex} / \mathrm{Em}}=405 / 450 \mathrm{~nm} ; \mathrm{FITC}, \lambda_{\mathrm{Ex} / \mathrm{Em}}=488 / 520 \mathrm{~nm}$ ).

B-4 was used (Fig. 6); images show clear improvements in the green emission localized in the intracellular regions. These results indicate that $\mathrm{CB}[7]$ complexation is retained in vitro with a preserved inhibition of nonradiative decay pathways for B-4. More investigations are necessary to determine what are the mechanisms of cell uptake and whether the host and the guest are internalized together or separately. However, these experiments exceed the scope of this study. Nevertheless these results illustrate well the potential of $\mathrm{CB}[7]$ to improve the fluorescence properties of a dye, even by external complexation.

\section{Conclusions}

In summary, we describe the synthesis of three water soluble cationic BODIPY derivatives (B-4, B-5, and B-6) and the effect of $\mathrm{CB}$ [7] complexation on their photophysical properties. UV-vis absorption and fluorescence measurements have shown that these BODIPYs do not aggregate in water in the concentration range studied and are weakly emissive mainly due to charge transfer for B-4 and heavy atom effect for B-5 and B-6. However, the addition of $\mathrm{CB}[7]$ improved guest fluorescence by $\sim 10$ fold for B-4 and by $\sim 3$ fold for B-5, while B-6 remained essentially non-emissive in the solution and in the solid state. Fluorescence lifetimes were observed to be increased from 0.18 to $6.21 \mathrm{~ns}$ for B-4 and from 0.60 to $3.84 \mathrm{~ns}$ for B-5 after $\mathrm{CB}[7]$ addition. The origin of these improved photophysical properties upon $\mathrm{CB}[7]$ binding was studied by (TD)DFT and assigned to (i) the formation of external BODIPY.CB[7] complexes increasing the energy of the CT state for B-4 and B-5 as a result of ion-dipole interactions between the guest pyridinium and the carbonyl groups of the host favoring radiative decay from the locally-excited (LE) $\pi \rightarrow \pi^{*}$ transition state of BODIPY and (ii) the restrictions of rotational and vibrational motions upon host binding. With a preserved singlet oxygen generation, $\mathrm{CB}[7]$ complexation was shown to selectively inhibit CT but not ISC of BODIPYs in solution, in the solid state, and in vitro. These improvements allowed to use the BODIPY.CB[7] complexes for confocal fluorescence imaging of U87 cells. While B-4, B-5, and B-6 alone only showed marginal fluorescence in vitro, clear emission from the intracellular regions was evidenced after co-incubation with B-4 and $\mathrm{CB}[7]$, supporting the possible use of external cucurbituril complexes for fluorescence imaging. Pleasingly, singlet oxygen generation was shown to remain unaffected by $\mathrm{CB}[7]$ complexation for B-5 and B-6 supporting possible use for photodynamic therapy (PDT). These results suggest that new BODIPY. $\mathrm{CB}[n]$ complexes could be designed for combined applications like simultaneous fluorescence imaging and PDT in a theragnostic context.

\section{Conflicts of interest}

There are no conflicts to declare.

\section{Acknowledgements}

CNRS and Aix Marseille University are acknowledged for providing continuous support.

\section{Notes and references}

1 H. Klfout, A. Stewart, M. Elkhalifa and H. He, ACS Appl. Mater. Interfaces, 2017, 9, 39873-39889.

2 A. Kamkaew, S. H. Lim, H. B. Lee, L. V. Kiew, L. Y. Chung and K. Burgess, Chem. Soc. Rev., 2013, 42, 77-88.

3 X. Cui, C. Zhang, K. Xu and J. Zhao, J. Mater. Chem. C, 2015, 3, 8735-8759.

4 N. Boens, V. Leen and W. Dehaen, Chem. Soc. Rev., 2012, 41, 1130-1172.

5 C. L. Liu, Y. Chen, D. P. Shelar, C. Li, G. Cheng and W. F. Fu, J. Mater. Chem. C, 2014, 2, 5471-5478.

6 E. Ozcan, M. Ozdemir, D. Ho, Y. Zorlu, R. Ozdemir, C. Kim, H. Usta and B. Cosut, ChemPlusChem, 2019, 84, 1423-1431.

7 D. Pfeifer, A. Russegger, I. Klimant and S. M. Borisov, Sens. Actuators, B, 2020, 304, 127312.

8 J. M. Franke, B. K. Raliski, S. C. Boggess, D. V. Natesan, E. T. Koretsky, P. Zhang, R. U. Kulkarni, P. E. Deal and E. W. Miller, J. Am. Chem. Soc., 2019, 141, 12824-12831.

9 P. Kaur and K. Singh, J. Mater. Chem. C, 2019, 7, 11361-11405.

10 L. Wang, L. Li and D. Cao, Sens. Actuators, B, 2017, 239, 1307-1317.

11 S. Kim, J. Bouffard and Y. Kim, Chem. - Eur. J., 2015, 21, 17459-17465.

12 H. Lu, Q. Wang, L. Gai, Z. Li, Y. Deng, X. Xiao, G. Lai and Z. Shen, Chem. - Eur. J., 2012, 18, 7852-7861.

13 D. Tian, F. Qi, H. Ma, X. Wang, Y. Pan, R. Chen, Z. Shen, Z. Liu, L. Huang and W. Huang, Nat. Commun., 2018, 9, 1-9. 14 S. Choi, J. Bouffard and Y. Kim, Chem. Sci., 2014, 5, 751-755. 15 S. Xu, W. Wu, X. Cai, C. J. Zhang, Y. Yuan, J. Liang, G. Feng, P. Manghnani and B. Liu, Chem. Commun., 2017, 53, 8727-8730.

16 Z. Liu, Z. Jiang, M. Yan and X. Wang, Front. Chem., 2019, 7, $1-16$. 
17 E. Özcan, B. Dedeoglu, Y. Chumakov, A. G. Gürek, Y. Zorlu, B. Çoşut and M. Menaf Ayhan, Chem. - Eur. J., 2021, 27, 1603-1608.

18 C. Chen, X. Ni, H. W. Tian, Q. Liu, D. S. Guo and D. Ding, Angew. Chem., Int. Ed., 2020, 59, 10008-10012.

19 X. Y. Lou, N. Song and Y. W. Yang, Chem. - Eur. J., 2019, 25, 11975-11982.

20 X. Yao, T. Li, J. Wang, X. Ma and H. Tian, Adv. Opt. Mater., 2016, 4, 1322-1349.

21 R. N. Dsouza, U. Pischel and W. M. Nau, Chem. Rev., 2011, 111, 7941-7980.

22 F. Schibilla, L. Stegemann, C. A. Strassert, F. Rizzo and B. J. Ravoo, Photochem. Photobiol. Sci., 2016, 15, 235-243.

23 Y. L. Loukas, Analyst, 1997, 122, 377-381.

24 J. W. Lee, S. Samal, N. Selvapalam, H. J. Kim and K. Kim, Acc. Chem. Res., 2003, 36, 621-630.

25 K. I. Assaf and W. M. Nau, Chem. Soc. Rev., 2015, 44, 394-418. 26 J. Lagona, P. Mukhopadhyay, S. Chakrabarti and L. Isaacs, Angew. Chem., Int. Ed., 2005, 44, 4844-4870.

27 O. Reany, A. Li, M. Yefet, M. K. Gilson and E. Keinan, J. Am. Chem. Soc., 2017, 139, 8138-8145.

28 V. D. Uzunova, C. Cullinane, K. Brix, W. M. Nau and A. I. Day, Org. Biomol. Chem., 2010, 8, 2037-2042.

29 D. Das, K. I. Assaf and W. M. Nau, Front. Chem., 2019, 7, 1-23.

30 M. Sayed and H. Pal, J. Mater. Chem. C, 2016, 4, 2685-2706. 31 H. Yin, F. Dumur, Y. Niu, M. M. Ayhan, O. Grauby, W. Liu, C. Wang, D. Siri, R. Rosas, A. Tonetto, D. Gigmes, R. Wang, D. Bardelang and O. Ouari, ACS Appl. Mater. Interfaces, 2017, 9, 33220-33228.

32 S. R. Wang, J. Q. Wang, G. H. Xu, L. Wei, B. S. Fu, L. Y. Wu, Y. Y. Song, X. R. Yang, C. Li, S. M. Liu and X. Zhou, Adv. Sci., 2018, 5, 1-9.

33 C. Hu, L. Grimm, A. Prabodh, A. Baksi, A. Siennicka, P. A. Levkin, M. M. Kappes and F. Biedermann, Chem. Sci., 2020, 11, 11142-11153.

34 X. Ma and Y. Zhao, Chem. Rev., 2015, 115, 7794-7839.

35 A. Palma, M. Artelsmair, G. Wu, X. Lu, S. J. Barrow, N. Uddin, E. Rosta, E. Masson and O. A. Scherman, Angew. Chem., Int. Ed., 2017, 56, 15688-15692.

36 Z. Zeng, X. Zhang, G. Luo, Y. Meng, L. Zhang, W. Zhao, Z. Tao and Q. Zhang, J. Mater. Chem. C, 2021, 9, 6160-6165.
37 X. Zhang, X. Xu, S. Li, L. H. Wang, J. Zhang and R. Wang, Sci. Rep., 2018, 8, 1-7.

38 W. M. Nau and J. Mohanty, Int. J. Photoenergy, 2005, 7, 133-141.

39 S. S. Thakare, G. Chakraborty, P. Krishnakumar, A. K. Ray, D. K. Maity, H. Pal and N. Sekar, J. Phys. Chem. B, 2016, 120, 11266-11278.

40 B. Yuan, H. Wu, H. Wang, B. Tang, J. F. Xu and X. Zhang, Angew. Chem., Int. Ed., 2021, 60, 706-710.

41 J. F. Yin, Y. Hu, D. G. Wang, L. Yang, Z. Jin, Y. Zhang and G. C. Kuang, ACS Macro Lett., 2017, 6, 139-143.

42 M. A. Alnajjar, J. Bartelmeß, R. Hein, P. Ashokkumar, M. Nilam, W. M. Nau, K. Rurack and A. Hennig, Beilstein J. Org. Chem., 2018, 14, 1961-1971.

43 M. Gupta, K. Parvathi, S. Mula, D. K. Maity and A. K. Ray, Photochem. Photobiol. Sci., 2017, 16, 499-506.

44 O. Buyukcakir, F. T. Yasar, O. A. Bozdemir, B. Icli and E. U. Akkaya, Org. Lett., 2013, 15, 1012-1015.

45 A. Singh, W. T. Yip and R. L. Halterman, Org. Lett., 2012, 14, 4046-4049.

46 G. Chakraborty, M. K. Choudhary, M. Sundararajan, A. K. Ray, S. Mula and H. Pal, J. Phys. Chem. B, 2021, 125, 7946-7957.

47 M. M. Ayhan, E. Özcan, B. Dedeoglu, Y. Chumakov, Y. Zorlu and B. Coşut, CrystEngComm, 2021, 23, 268-272.

48 J. Karolin, L. B. A. Johansson, L. Strandberg and T. Ny, J. Am. Chem. Soc., 1994, 116, 7801-7806.

49 A. Gorman, J. Killoran, C. O’Shea, T. Kenna, W. M. Gallagher and D. F. O'Shea, J. Am. Chem. Soc., 2004, 126, 10619-10631.

50 X. Miao, W. Hu, T. He, H. Tao, Q. Wang, R. Chen, L. Jin, H. Zhao, X. Lu, Q. Fan and W. Huang, Chem. Sci., 2019, 10, 3096-3102.

51 I. W. Wyman and D. H. MacArtney, Org. Biomol. Chem., 2008, 6, 1796-1801.

52 D. Bardelang, J. L. Clément, J. P. Finet, H. Karoui and P. Tordo, J. Phys. Chem. B, 2004, 108, 8054-8061.

53 Z. Lin, A. W. Kohn and T. Van Voorhis, J. Phys. Chem. C, 2020, 124, 3925-3938.

54 R. P. Sabatini, T. M. McCormick, T. Lazarides, K. C. Wilson, R. Eisenberg and D. W. McCamant, J. Phys. Chem. Lett., 2011, 2, 223-227. 